\title{
Development of a visco-elastoplastic contact force model and its parameter determination for apples
}

\author{
Elien Diels ${ }^{\mathrm{a}, *}$, Tim Odenthal $^{\mathrm{a}, \mathrm{b}}$, Janos Keresztes $^{\mathrm{a}}$, Simon Vanmaercke ${ }^{\mathrm{a}}$, Pieter Verboven ${ }^{\mathrm{a}}$, \\ Bart Nicolaï $^{\mathrm{a}}$, Wouter Saeys ${ }^{\mathrm{a}}$, Herman Ramon ${ }^{\mathrm{a}}$, Bart Smeets ${ }^{\mathrm{a}, \mathrm{b}}$ \\ ${ }^{a}$ KU Leuven-MeBioS, Kasteelpark Arenberg 30, 3001 Heverlee, Belgium \\ ${ }^{\mathrm{b}} \mathrm{KU}$ Leuven-Biomechanics Section, Celestijnenlaan 300c, 3001 Heverlee, Belgium
}

\section{A R T I C L E I N F O}

\section{Article history:}

Received 20 January 2016

Received in revised form 3 June 2016

Accepted 6 June 2016

Available online $\mathrm{xxx}$

\section{Keywords:}

Apple

Discrete Element Method

Normal contact force model

Mechanical properties

\begin{abstract}
A B S T R A C T
A contact force model was developed to model the visco-elastoplastic (VEP) behaviour of apples. The model is based on the elastoplastic Thornton model and has been written in a pressure-based formulation to extend the application of the model to Discrete Element Method (DEM) simulations with arbitrary rounded shapes. The parameters of the new developed VEP contact force model were determined by fitting the experimental data acquired from Jonagold, Joly Red and Kanzi apples impacted by a pendulum. With only one parameter set per cultivar and for a large impact range (impact velocity range: $0.3-1.5 \mathrm{~m} / \mathrm{s})$, the VEP-model $\left(\mathrm{R}^{2}=0.90 \pm 0.13\right)$ provides a better description of the forcedeformation profiles than the viscoelastic Kono and Kuwabara $(K K)$ model $\left(R^{2}=0.71 \pm 0.20\right)$. The equivalent Young's modulus $\left(\mathrm{E}^{*}\right)$ was also determined under quasi-static conditions, which resulted in measured $E^{*}$-values for Jonagold, Joly Red and Kanzi apples of respectively $4.24 \pm 0.96 \mathrm{MPa}, 5.09 \pm 1.27$ $\mathrm{MPa}$ and $7.82 \pm 0.41 \mathrm{MPa}$. The novel VEP-model has the potential to help predict and understand bruise damage in apples as well as other horticultural products.
\end{abstract}

(c) 2016 Elsevier B.V. All rights reserved.

\section{Introduction}

For apples, bruising is the major postharvest mechanical damage problem (Knee and Miller, 2002; Van Zeebroeck et al., 2006). In apples, bruising may occur in several ways. On the tree, apples can bruise by colliding with branches or other apples. At harvest, pickers can damage the apple by applying too high force with the fingers or by dropping the apple into the picking baskets. On their way to the consumer, apples bruise due to the vibrations that occur during the various forms of transport (e.g. fork-lift tractor) and due to impact against other apples or hard surfaces during grading and container transfers. Furthermore, bruise damage may also result from consumer handling in store (rifling) or compression inflicted by the neighbouring apples during transport and storage (Knee and Miller, 2002). Dynamic forces during transport and handling are the main causes of bruise damage (Mohsenin, 1986; Van Zeebroeck et al., 2006). Simulations

\footnotetext{
* Corresponding author.

E-mail address: elien.diels@biw.kuleuven.be (E. Diels).
}

that can model both the kinematics, dynamics and the resulting bruise damage of apples during transport and handling could help to optimise sorting lines and packaging materials to reduce the likelihood of bruise damage. The Discrete Element Method (DEM) is a suitable numerical technique to perform such simulations (Tijskens et al., 2003). Recently, a new method for modelling arbitrary shapes in DEM has been developed (Smeets et al., 2014), which makes DEM more suitable for modelling fruit by taking into account their non-perfectly-spherical shape. Here, interaction forces are not computed directly, but obtained by numerically integrating the pressure over the contact area between two particles. The latter is important for modelling damage, since bruise damage is related to local pressure levels.

However, a normal contact force model (i.e. a model that relates the normal component of the deformation vector to the normal component of the contact force) that adequately describes both viscoelastic and plastic deformations is still lacking. Apple tissue behaves visco-elastoplastically when the contact pressure exceeds a critical value (Van Zeebroeck, 2005). As yet, the viscoelastic Kuwabara and Kono (KK) model (Brilliantov et al., 1996a,b; Kuwabara and Kono, 1987; Van Zeebroeck, 2005) has been 
commonly used to describe contact behaviour of fruit and vegetables (Van Zeebroeck, 2005; Ahmadi et al., 2012). This viscoelastic KK-model generalizes Hertz' law to include viscous damping forces that occur during contact between viscoelastic bodies. The KK-model states the following relation for the contact force between particles with radius of curvature $R_{i}(i=1,2)$ :

$F^{K K}=\frac{4}{3} E^{*} \sqrt{R^{*}}\left(\delta^{\frac{3}{2}}+A^{*} \delta \sqrt{\delta}\right)$

where $\delta$ is the deformation (i.e. the apparent overlap), $\dot{\delta}$ is the relative normal velocity between the two contacting particles, $A^{*}$ is the equivalent dissipative parameter combining the viscous properties of the two colliding bodies, $R^{*}$ is the effective radius of curvature and $E^{*}$ is the equivalent Young's modulus which are defined as:

$A^{*}=\frac{A_{1}+A_{2}}{2}, R^{*}=\left(\frac{1}{R_{1}}+\frac{1}{R_{2}}\right)^{-1}$ and $\frac{1}{E^{*}}=\frac{1-v_{1}^{2}}{E_{1}}+\frac{1-v_{2}^{2}}{E_{2}}$

with $E_{i}$ and $v_{i}$ respectively the Young's modulus and the Poisson's ratio of one of the impacting objects.

The total Hertz pressure that includes the elastic Hertzian pressure $\left(p_{e}\right)$ and the dissipative pressure $\left(p_{v}\right)$ associated with the damping, can be written as (Brilliantov et al., 1996a):

$$
\begin{aligned}
p^{K K}(r) & =p_{v}(r)+p_{e}(r), \text { with } p_{v}(r) \\
& =\frac{E^{*}}{\pi} \sqrt{R^{*} \delta-r^{2}} \frac{3 A^{*} \delta}{R^{*} \delta} \text { and } p_{e}(r)=\frac{2 E^{*}}{R^{*} \pi} \sqrt{R^{*} \delta-r^{2}}
\end{aligned}
$$

The contact parameters of the KK-model have been determined for apples, potatoes, tomatoes and peaches, using a non-linear least square fitting technique on loading data obtained by a pendulum device (Van Zeebroeck, 2005; Ahmadi et al., 2012). Van Zeebroeck (2005) argued that this model can also describe an impact where plastic energy dissipation occurs if the contact force model parameters are allowed to depend on the degree of impact. His justification is that the net effect of both viscous and plastic energy dissipation is captured by the damping term.

Several elastoplastic models have been developed (KruggelEmden et al., 2009; Rathbone et al., 2015; Thornton and Ning, 1998; Vu-Quoc et al., 2000; Zhang and Vu-Quoc, 2002). The elastoplastic Thornton contact force model consists of a Hertzian unloading phase and a loading phase comprising a Hertzian elastic part followed by a linear plastic part. The Thornton pressure during

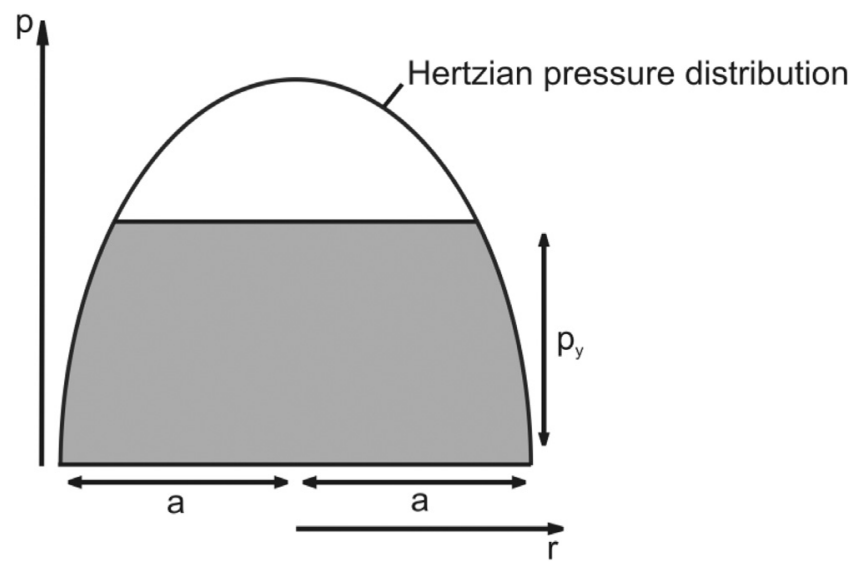

Fig. 1. The pressure distribution assumed by the Thornton model over the contact area with radius $a$ during the loading phase in which plastic deformation occurs (indicated in grey). loading $\left(p_{\text {load }}^{T}\right)$ is depicted in Fig. 1 and is expressed as (Thornton and Ning, 1998):

$$
p_{\text {load }}^{T}(r)=\left\{\begin{array}{cc}
p_{e}(r) & \delta<\delta_{y}, \dot{\delta}>0 \\
p_{y} & \delta \geq \delta_{y}, \dot{\delta}>0
\end{array}\right.
$$

where $p_{y}$ is the yield pressure and $\delta_{y}$ is the deformation above which plastic deformation starts to occur. Since, according to this model, the pressure is purely elastic until plastic deformation takes place, $\delta_{y}$ is defined as:

$\delta_{y}=\frac{p_{y}^{2} \pi^{2} R^{*}}{4 E^{*}}$

In the work of Thornton and Ning (1998), the Thornton force $\left(F^{T}\right)$ during loading was derived via integration of $p_{\text {load }}^{T}$ (Eq. (4)). The net Thornton force during unloading follows from the assumption of a Hertzian unloading phase with a reduced curvature corresponding to the point of maximum compression. For a detailed derivation thereof the Thornton force we refer to the work of Thornton and Ning (1998). The force-deformation relationship of the Thornton model is expressed as:

$F^{T}=\left\{\begin{array}{ll}\frac{4}{3} E^{*} \sqrt{R^{*}} \frac{3}{2} & \delta<\delta_{y}, \dot{\delta}>0 \\ 2 E^{*} \sqrt{R^{*} \delta_{y}}\left(\delta-\delta_{y}\right)+\frac{4}{3} E^{*} \sqrt{R^{*}} \delta_{y}^{\frac{3}{2}} & \delta>\delta_{y}, \dot{\delta}>0 \\ \frac{4}{3} E^{*} \sqrt{R_{p}^{*}}\left(\delta+R^{*} \frac{\delta_{\max }}{R_{p}^{*}}-\delta_{\max }\right)^{3 / 2} & \dot{\delta}<0\end{array}\right\}$

where $R_{p}^{*}$ is the corrected effective radius of curvature expressed as:

$R_{p}^{*}=\frac{4 E^{*}}{3 F_{\max }}\left(\frac{2 F_{\max }+F_{y}}{2 \pi p_{y}}\right)^{3 / 2}$

with $F_{\max }$ the maximum force that occurs at a maximum deformation $\delta_{\max }$ at the end of the loading phase.

The main objective of this study was to enable realistic DEMsimulations with apples, wherein bruise damage can be predicted, hypothesizing that bruising occurs above a certain pressure (yield pressure) threshold. Support for this hypothesis comes from the fact that bruise damage is characterised by the failure of cells on which the stress exceeds a critical value (Baritelle and Hyde, 2001). Modelling bruises based on more simple mechanical measures such as force, impact energy, etc. will lead to less successful predictions since such models neglect the importance of the geometry (i.e. contact area, curvature, etc.) in the problem. To clarify the latter, although the peak force and/or impact energy might be the same in case of an impact in a standardised test and an impact in the apple handling chain, the pressure distribution in both cases may vary significantly from each other since the shape and material properties of the object(s) that come into contact with the apple can differ. Since apple tissue behaves viscoelastoplastically and bruising is related to pressure, an important step towards realistic simulations is the development of a viscoelastoplastic (VEP) model with which the pressure evolution in apple tissue can be computed and which can be used to determine the yield pressure of apples. In this study a VEP model was elaborated that is based on both the KK-model and the Thornton model.

First, the visco-elastoplastic contact force model is introduced and the proposed pressure formulation is related to its force-based equivalent. Next, the experimental set-up and data-processing of the quasi-static and dynamic measurements are described and the model performance is evaluated on experimental data obtained on 
apples. Finally, the limitations of the use of the VEP-model to predict bruise damage are discussed.

\section{Materials and methods}

2.1. Visco-elastoplastic contact model: force- and pressure-based formulation

A visco-elastoplastic variant of the elastoplastic Thornton model was created by adding the dissipative force term of the KK-model to the Thornton force. The force-deformation relationship of the VEP-model is expressed as:

$$
F^{V E P}=\left\{\begin{array}{ll}
\frac{4}{3} E^{*} \sqrt{R^{*}}\left(\delta^{\frac{3}{2}}+A^{*} \dot{\delta} \sqrt{\delta}\right) & \delta<\delta_{y}, \dot{\delta}>0 \\
2 E^{*} \sqrt{R^{*} \delta_{y}}\left(\delta-\delta_{y}\right)+\frac{4}{3} E^{*} \sqrt{R^{*}} \delta_{y}^{\frac{3}{2}}+\frac{4}{3} E^{*} \sqrt{R^{*}} A^{*} \dot{\delta} \sqrt{\delta} & \delta>\delta_{y}, \dot{\delta}>0 \\
\frac{4}{3} E^{*} \sqrt{R_{p}^{*}}\left(\delta+R^{*} \frac{\delta_{\max }}{R_{p}^{*}}-\delta_{\max }\right)^{3 / 2}+\frac{4}{3} E^{*} \sqrt{R^{*}} A^{*} \dot{\delta} \sqrt{\delta} & \dot{\delta}<0
\end{array}\right\}
$$

A pressure-based formulation of the Thornton model and the visco-elastoplastic model was developed to implement the models in DEM for arbitrary shapes (Smeets et al., 2014). Contrary to the unloading phase, the pressure occurring during the loading phase is fully described by Thornton and Ning (1998) (see Eq. (4)). During unloading, the Thornton-pressure $p_{\text {unload }}^{T}(r)$ decreases in a Hertzian trend, parallel to a hypothetic purely elastic loading curve which extends to the maximal deformation $\delta_{\max }$, but which is offset by the difference between the maximal pressure in the Hertz regime $p_{\text {max }}(r)$ and the yield pressure. Since unloading is dependent on the specific maximum pressure $p_{\max }(r)$ that was reached during the given contact event, the contact state must in theory remember the maximal pressure for each evaluation (i.e. quadrature point) during a contact event. This approach is unfeasible, not only because it is computationally cumbersome, but also because the relative locations of the quadrature points are not conserved over time for a given contact. The maximum pressure $p_{\max }^{T}$ can, however, be approximated by making use of the maximal historical overlap $\left(\delta_{\max }^{\text {sphere }}\right)$ during a contact at the local curvature, and then back-computing $p_{\max }$ from the maximal overlap at a given $r$ as:

$p_{\max }^{T}(r)=\frac{2 E^{*}}{\pi R^{*}} \sqrt{R^{*} \delta_{\max }^{\text {sphere }}-r^{2}}$

where by $\delta_{\max }^{\text {sphere }}$ is calculated as the maximal virtual overlap distance of the local fitted sphere(s).

By performing simulations with a meshed sphere and a perfect sphere, it could be verified (see Appendix A) that Eq. (9) provides an excellent approximation of $p_{\max }$. The Thornton-pressure during unloading is then calculated as:

$$
p_{\text {unload }}^{T}(r)= \begin{cases}\max \left(0, p_{e}(r)-p_{\max }(r)+p_{y}\right) & p_{\max }^{T}(r)>p_{y} \\ p_{e}(r) & p_{\max }^{T}(r) \leq p_{y}\end{cases}
$$

The dissipative pressure term of the KK-model ( $p_{v}$ in Eq. (3)) was added to the pressure-based formulation of the Thornton model (Eqs. (4) and (10)) in order to obtain a visco-elastoplastic model. It should be noted that it is possible that the modelled maximal pressure becomes higher than the yield pressure, since the dissipative pressure is added to the Thornton pressure.

\subsection{Samples}

All the experiments were performed on apples that were picked at the end of September 2014 and stored under controlled atmosphere for 3.5 months. The Jonagold and Joly Red cultivar apples were stored at $1{ }^{\circ} \mathrm{C}, 1 \% \mathrm{O}_{2}$ and $3 \% \mathrm{CO}_{2}$, while apples of the Kanzi cultivar were stored at $4{ }^{\circ} \mathrm{C}, 2 \% \mathrm{O}_{2}$ and $0.7 \% \mathrm{CO}_{2}$.

\subsection{Quasi-static measurements of the equivalent Young's modulus}

All the normal contact force models described in this work contain the equivalent Young's modulus $E^{*}$. The experiment that was performed to obtain $E^{*}$ under quasi-static conditions makes use of a Universal Testing System (UTS Test Systeme, GmbH, Germany) equipped with a load cell (Wagezelle, HBM, Darmstadt, Germany) with a saturation force of $200 \mathrm{~N}$ and a reported sensitivity of $0.02 \mathrm{~N}$. Several experiments to measure $E^{*}$ on biological materials of convex shape have been described in literature (Arnold and Mohsenin, 1971; ASAE Standard, 1998). In this experiment, apples were cut in half and compressed between two parallel flat plates at a velocity of $0.04 \mathrm{~mm} / \mathrm{s}$ (Fig. 2). By compressing half apples instead of whole apples, only one contact point has to be considered, since the deformation at the bottom plate will be negligible (ASAE Standard, 1998). This simplifies the data-analysis and leads to more consistent results. Ten half apples were measured per cultivar. A first approximation of the beginning of contact was determined as the point in time where the force became larger than $0.2 \mathrm{~N}$. The deformation at this point was set to zero. $E^{*}$ was estimated by fitting Hertz' law (Eq. (1) with $A^{*}=0 \mathrm{~s}$ ) on the initial part of the force versus deformation curve of the loading phase (i.e. the part before a decrease in force occurs). To correct for errors made in the estimation of the beginning of contact, apart from parameter $E^{*}$, a second parameter was estimated, namely an offset that was added to the deformation in the Hertz' law. The radius of curvature of the apple at the point of compression was measured before compression with a height meter (Mitutoyo Belgium NV, Kruibeke, Belgium). The harmonic average was used to compensate for non-sphericity (Zarifneshat et al., 2010):

$R=\frac{2 r_{x} r_{y}}{r_{x}+r_{y}}$

where $r_{x}$ and $r_{y}$ are the radii of curvature measured in the direction of the stem-calyx axis and the axis perpendicular to the stem-calyx axis.

\subsection{Dynamic measurements}

\subsubsection{Experimental design}

The pendulum device depicted in Fig. 3 was used to impact half apples. Apart from a few minor changes, this is the same pendulum that was used by Van Zeebroeck (2005). The pendulum rod is released by an electro-magnet in order to obtain consistent

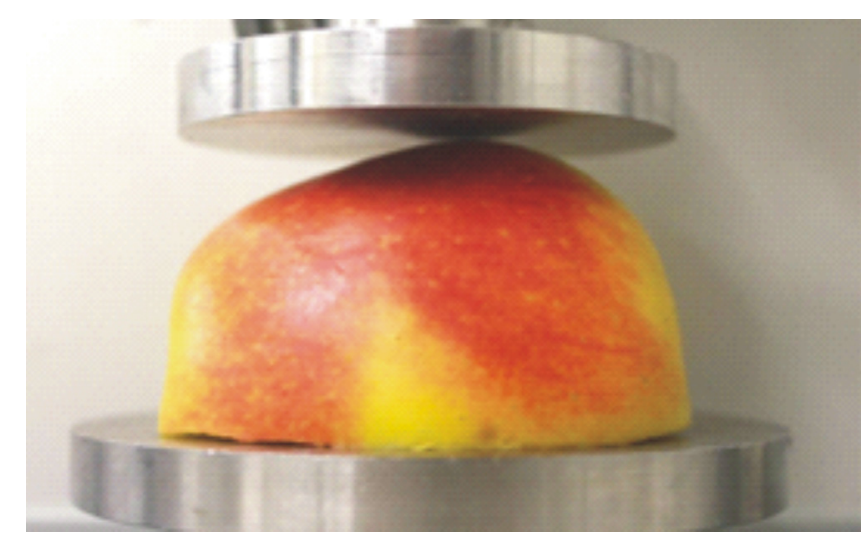

Fig. 2. Quasi-static compression experiment on half apples. 
(a)

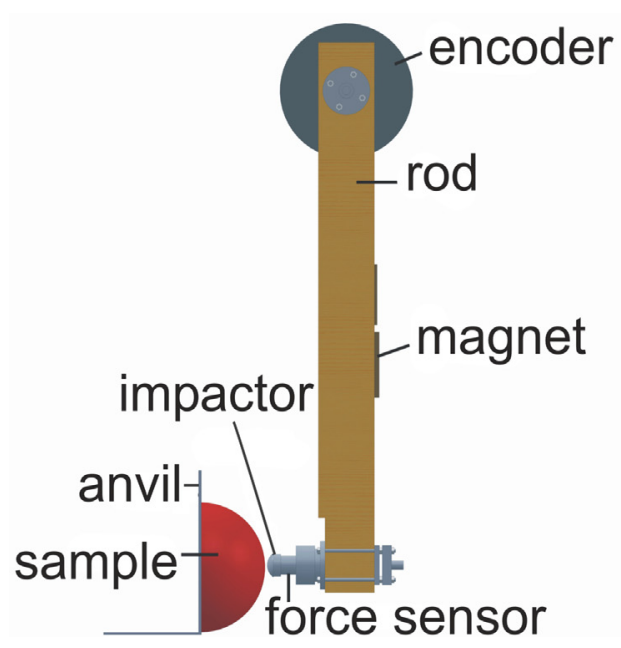

(b)

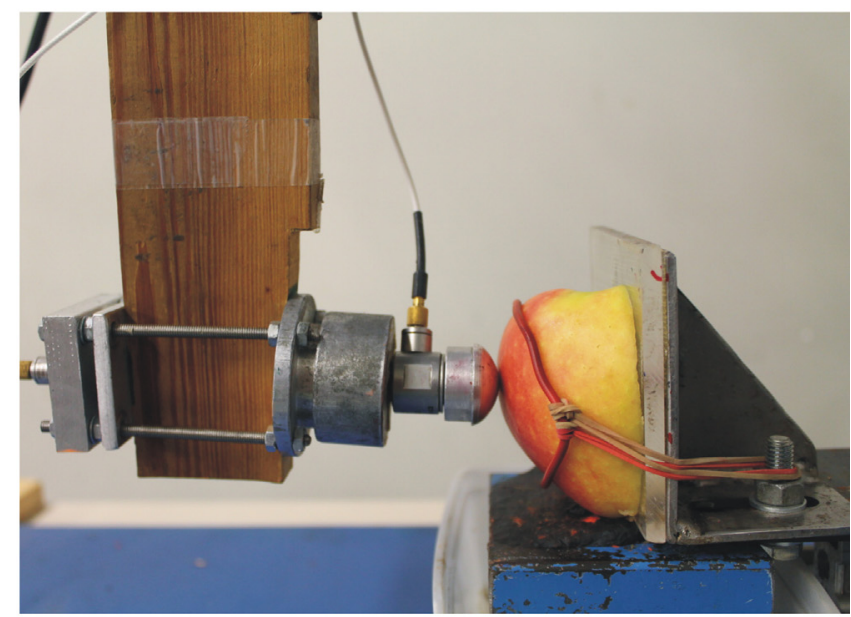

Fig. 3. (a) Schematic representation of the pendulum device. (b) Set-up of the dynamic experiment on half apples.

measurements. The anvil, on which the sample is mounted, is made rigid such that its deflection during impact can be neglected. The moment of inertia and the centre of gravity of the pendulum were determined by modelling the pendulum rod in SolidWorks (SolidWorks 2014, Dassault Systèmes SOLIDWORKS Corp., VélizyVillacoublay, France). The mass of each part of the pendulum rod was measured, such that the correct densities could be used. The obtained mass moment of inertia is $0.097 \mathrm{~kg} \mathrm{~m}^{2}$ and the centre of gravity is positioned at a distance of $0.297 \mathrm{~m}$ from the centre of rotation. The pendulum rod has a total mass of $0.776 \mathrm{~kg}$ and has an aluminium spherical impactor at its tip (radius of curvature: $0.015 \mathrm{~m}$ ). This impactor is mounted on a force sensor (Dytran 1051V3, Dytran instruments, Chatsworth, California, US; sensitivity: $11.3 \mathrm{mV} / \mathrm{N}$ ) that measures the force normal to the contact surface. The change in angle of the pendulum rod from the release position is measured by an incremental optical encoder (RON 275TTLx5, Heidenhain, Traunreut, Germany). The force and angle data was further processed in Matlab (R2015a, The MathWorks Inc., Nattick, MA). The angle data (in radians) was smoothed with a Savitzky-Golay filter (polynomial order 5, window length $0.01 \mathrm{~s}$ ) and multiplied by the distance from the centre of rotation to the tip of the impactor $(0.495 \mathrm{~m})$ to obtain the tangential displacement of the impactor. The deformation $\delta$ (i.e. indentation) of the specimen could be calculated by subtracting the tangential displacement at the beginning of contact from the instantaneous tangential displacement. The displacement rate during impact $\dot{\delta}$ was calculated as follows:

$\dot{\delta}(t)=\frac{f}{2}(\delta(t+1)+\delta(t-1))$

where $f$ is the sampling frequency $(200 \mathrm{kHz})$.

Per cultivar, forty half apples were impacted once by the pendulum with the spherical impactor (Fig. 3). Nine initial release angles of the pendulum rod were used, leading to impact velocities between $0.12 \mathrm{~m} / \mathrm{s}$ and $1.5 \mathrm{~m} / \mathrm{s}$ and impact energies (calculated as the area under the loading curves) between $0.003 \mathrm{~J}$ and $0.338 \mathrm{~J}$ (see Appendix B for an overview of the different impact levels). Just as in the UTS experiment, the curvature at the point of impact was measured with a Mitutoyo ${ }^{\circledR}$ height meter. The measured force and displacement were used to estimate the contact parameters of the KK-model, Thornton model and VEP-model.

\subsubsection{Contact model parameter estimation}

First, the parameter estimation to obtain the contact model parameters of the viscoelastic KK-model is explained. For each half apple, values for the equivalent dissipative parameter $A^{*}$ and the equivalent Young's modulus $E^{*}$ were estimated. This was done by fitting the measured force to the modelled force, whereby the measured deformation and deformation rate were used as independent variables. This non-linear fitting procedure was performed in Matlab based on the Trust-region-Reflective algorithm (Coleman and Li, 1996). After this parameter estimation was performed, linear regression equations were built with impact velocity as independent variable and the contact model parameters as dependent variables. At last, the previously described parameter estimation procedure was repeated on all the measurements of one cultivar at once, yielding one parameter set per cultivar.

To obtain the parameters of the Thornton contact force model, the previously described fitting procedure was carried out on all measurements of one cultivar at once. During this fitting procedure, the equivalent Young's modulus $E^{*}$ was fixed to the value found in the UTS experiment on half apples. Thereafter, the absorbed energy $\left(E_{a b s}\right)$ of the modelled and the measured curves were compared with each other, where $E_{a b s}$ was calculated as the area between the loading and unloading curve.

The fitting procedure for the VEP-model was also carried out on all the measurements of one cultivar at once. The equivalent Young's modulus $E^{*}$ was again fixed to the value estimated from the UTS-experiment and the viscous damping in the loading phase $\left(A_{\text {load }}^{*}\right)$ was allowed to differ from the one in the unloading phase $\left(A_{\text {unload }}^{*}\right)$. The justification for the latter is that the viscosity of intact apple tissue may in general be different from the viscosity of bruised apple tissue.

\section{Results and discussion}

\subsection{Quasi-static loading experiment}

The quasi-static loading curves in Fig. 4 show a sudden small decrease in force followed by a further increase in force. Such a decreasing force was visible in most of the measurements and was also visible in the typical loading curve reported by Herold et al. (2001). This decrease in force can most likely be explained by 
(a)

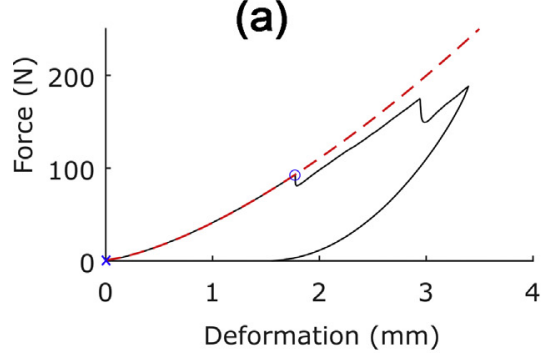

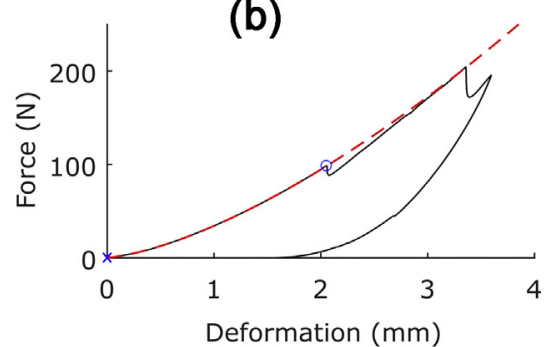

Deformation $(\mathrm{mm})$

$$
\begin{aligned}
\hline & \text { Measured } \\
\times & \text { Lower fitting boundary } \\
& \text { Upper fitting boundary } \\
- & - \text { - Fitted Hertz curve }
\end{aligned}
$$

Fig. 4. Hertz theory fitted on two UTS measurements of half apples where two failure events are visible.

initial cell damage. After the rupturing of a small volume of cells, further compression causes the contact area and the force to increase until the following layer(s) of cells rupture, causing a significant decrease in force. To ensure that no significant plastic material behaviour is present in the data used to fit the purely elastic Hertz' law, only the first part of the curve until the first drop in force was fitted-see Fig. 4. As can be observed in that figure, Hertz theory fits this part of the data very well (average $\mathrm{R}^{2}=0.995$ ). Further analysis of the force-deformation curves is given in Appendix C.

After the dip in force, the course of the loading curves stays more or less parallel to the fitted Hertzian curve in most of the measurements, and sometimes (e.g. Fig. 4b) even increases faster with increasing deformation. However, the Thornton model assumes a linear force increase in the plastic region such that the force starts to deviate from the faster increasing hypothetical Hertzian force curve. Since no linear part appears in the measured loading curves, the Thornton model does not allow to fit the force versus deformation curves well. However, the shape of the unloading curve of the Thornton model corresponds well to the observed shape.

Although the shape of the force versus deformation curve of the Thornton model cannot entirely match the shape of the measured curves, the assumption made by Thornton in the loading phase (i.e. that the pressure remains constant in the area where the hypothetical Hertzian pressure rises above the yield pressure) is probably not far from reality. Lewis et al. (2008) observed that the pressure, measured during static loading of an apple between two flat planes, was always higher in the centre and decreased towards the edges. For different loads, the maximum pressure remained around $0.5 \mathrm{MPa}$ and the area at this pressure level increased with increasing load (Lewis et al., 2008). This observation agrees with the assumption of Thornton in the loading phase, with a yield stress of $0.5 \mathrm{MPa}$.

\section{Table 1}

Average equivalent Young's modulus $\left(E^{*}\right)$ values obtained from the UTS-experiment on half apples and their corresponding standard deviations (Std) and 95\% confidence intervals $(\mathrm{CI})$ using the sample Std as the population mean.

\begin{tabular}{llll}
\hline & $E^{*}(\mathrm{MPa})$ & \\
\cline { 2 - 4 } & Mean & Std & $\mathrm{CI}$ \\
\hline Jonagold & 4.24 & 0.96 & {$[3.553 ; 4.927]$} \\
Joly Red & 5.09 & 1.27 & {$[4.182 ; 5.999]$} \\
Kanzi & 7.82 & 0.41 & {$[7.527 ; 8.113]$} \\
\hline
\end{tabular}

From Table 1 it can be observed that the highest Young's modulus was found for Kanzi apples, followed by Joly Red. A pairwise comparison by means of the Tukey procedure, using a family confidence coefficient of 95\%, showed that Kanzi apples are significantly stiffer than Jonagold and Joly Red apples ( $p=1.60 \mathrm{e}-8$ and $1.94 \mathrm{e}-6$ respectively). The sample size was not large enough to detect a significant difference in average stiffness between Jonagold and Joly Red apples $(\mathrm{p}=0.14)$. Arnold and Mohsenin (1971) performed flat plate loading tests on whole apples of the cultivar Golden Delicious and reported $E^{*}$ values between 2.8 and $6.8 \mathrm{MPa}$, corresponding well to the $E^{*}$ values presented in Table 1 . Furthermore, these measured $E^{*}$ values are close to the estimated $E^{*}$ values found by fitting the VEP-model to the impact data (see Appendix D).

\subsection{Dynamic loading experiment}

\subsubsection{Parameter estimation using the KK-model}

In Table 2 the linear regression equations are shown that predict the estimated contact parameters of the KK-model based on the impact velocity. It can be seen that $E^{*}$ decreases with increasing impact velocity. Van Zeebroeck (2005) found a similar relationship. However, in literature, contradicting results can be found concerning the effect of strain rate on the Young's modulus (Baritelle and Hyde, 2001).

In Fig. 5 the measured hysteresis curves of nine half apples are presented together with the corresponding fitted curves obtained with the KK-model whereby the $\mathrm{E}^{*}$ and $\mathrm{A}^{*}$-values were determined by substituting the measured impact velocity into the linear regression equations in Table 2 . As can be seen, the shape of the fitted curves differs significantly from the measured curves. Due to the overestimated damping term in the KK-model, the beginning of the load curve of the fitted hysteresis curve has a concave shape, whereas the measured curve is more convex at the beginning. To conclude, even when a parameter set is estimated exclusively on

\section{Table 2}

Linear regression equations of $E^{*}$ and $A^{*}$ as a function of impact velocity $\left(\dot{\delta}_{i m p}\right)$ in $\mathrm{m} / \mathrm{s}$ and their $\mathrm{R}^{2}$ values. $E^{*}$ and $A^{*}$ were estimated by fitting the KK-model to the measured force-deformation curves.

\begin{tabular}{lllll}
\hline & $E^{*}=$ & $\mathrm{R}^{2}$ & $A^{*}=$ & $\mathrm{R}^{2}$ \\
\hline Jonagold & $-1.70 \mathrm{e} 6 \dot{\delta}_{\text {imp }}+4.10 \mathrm{e} 6$ & 0.65 & $-6.19 \mathrm{e}-4 \dot{\delta}_{\text {imp }}+0.0033$ & 0.34 \\
Joly Red & $-1.38 \mathrm{e} 6 \dot{\delta}_{\text {imp }}+3.26 \mathrm{e} 6$ & 0.69 & $-8.79 \mathrm{e}-4 \dot{\delta}_{\text {imp }}+0.0038$ & 0.28 \\
Kanzi & $-2.68 \mathrm{e} 6 \dot{\delta}_{\text {imp }}+5.73 \mathrm{e} 6$ & 0.77 & $-2.81 \mathrm{e}-4 \dot{\delta}_{\text {imp }}+0.0031$ & 0.12 \\
\hline
\end{tabular}




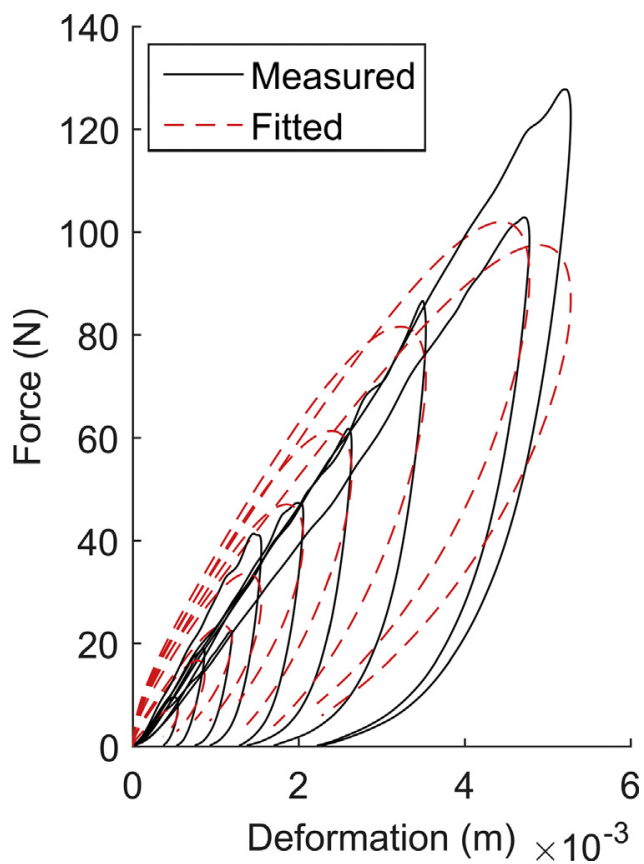

Fig. 5. Force versus deformation curves measured on Jonagold apples at nine different angles and impact velocities between 0.12 and $1.46 \mathrm{~m} / \mathrm{s}$ and the corresponding fitted curves using the KK-model $\left(\mathrm{E}^{*}\right.$ and $\mathrm{A}^{*}$-values were determined by substituting the measured $\dot{\delta}_{\text {imp }}$ into the linear regression equations in Table 2).

data of one half apple, the model does not succeed in fitting the data of that specific measurement. When the parameter estimation procedure was repeated on all the measurements of one cultivar at once, yielding one parameter set per cultivar, even worse fits were obtained (see Fig. 6a).

\subsubsection{Parameter estimation using elastoplastic Thornton model}

Van Zeebroeck (2005) asserted that it has never been verified whether applying the elastoplastic Thornton model would lead to unacceptable errors. In Fig. $6 \mathrm{~b}$, it is shown that the elastoplastic Thornton model underestimates the peak force and absorbed energy $\left(E_{a b s}\right)$. The relative absolute error in $E_{a b s}$ as a function of

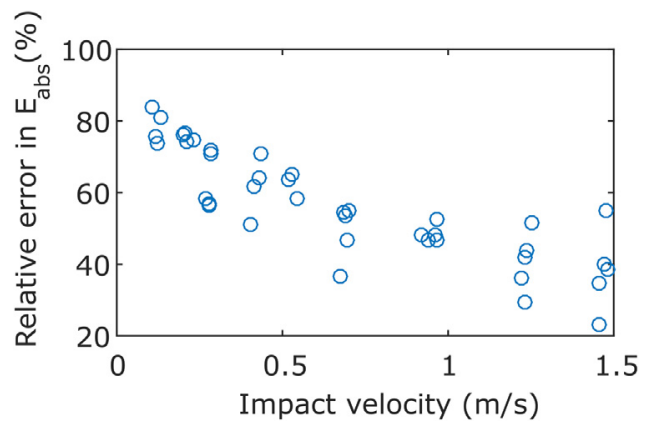

Fig. 7. For each dynamic measurement on Jonagold the absolute relative error in absorbed energy $\left(E_{a b s}\right)$ made by the Thornton model $\left(E^{*}=4.2 \mathrm{MPa}, p_{y}=0.63 \mathrm{MPa}\right)$ is displayed as a function of the impact velocity.

impact velocity (Fig. 7) shows that the Thornton model indeed underestimates the total absorbed energy. Especially for small impact velocities, where the majority of the total energy loss is due to viscous instead of plastic dissipation, the total absorbed energy is strongly underestimated. Thus, with a contact force model that uses realistic mechanical parameters, a dissipative term is necessary to obtain acceptable estimates for both force and absorbed energy.

\subsubsection{Parameter estimation using the VEP-model}

In Fig. 6c, it is shown that the shape of the measured curves corresponds well to the shape of the modelled curves using the VEP-model. However, the modelled unloading curves always look stiffer than the measured unloading. For low impact velocities, the VEP-model performed worse than the KK-model with velocity dependent parameters. At impact velocities lower than $0.3 \mathrm{~m} / \mathrm{s}$, the average of the $R^{2}$ values per measurement was respectively $0.51 \pm 0.22$ and $0.74 \pm 0.18$ for the VEP-model and the KK-model. Whereas at impact velocities higher than $0.3 \mathrm{~m} / \mathrm{s}, \mathrm{R}^{2}$ values of $0.90 \pm 0.13$ and $0.76 \pm 0.18$ were found for respectively the VEPmodel and the KK-model. However, the parameters of the VEPmodel were not allowed to vary with impact velocity whereas those of the KK-model were. In case also only one parameter set per cultivar was estimated for the KK-model, much worse fits were obtained ( $\mathrm{R}^{2}$ values of $0.11 \pm 0.20$ and $0.71 \pm 0.20$ for respectively
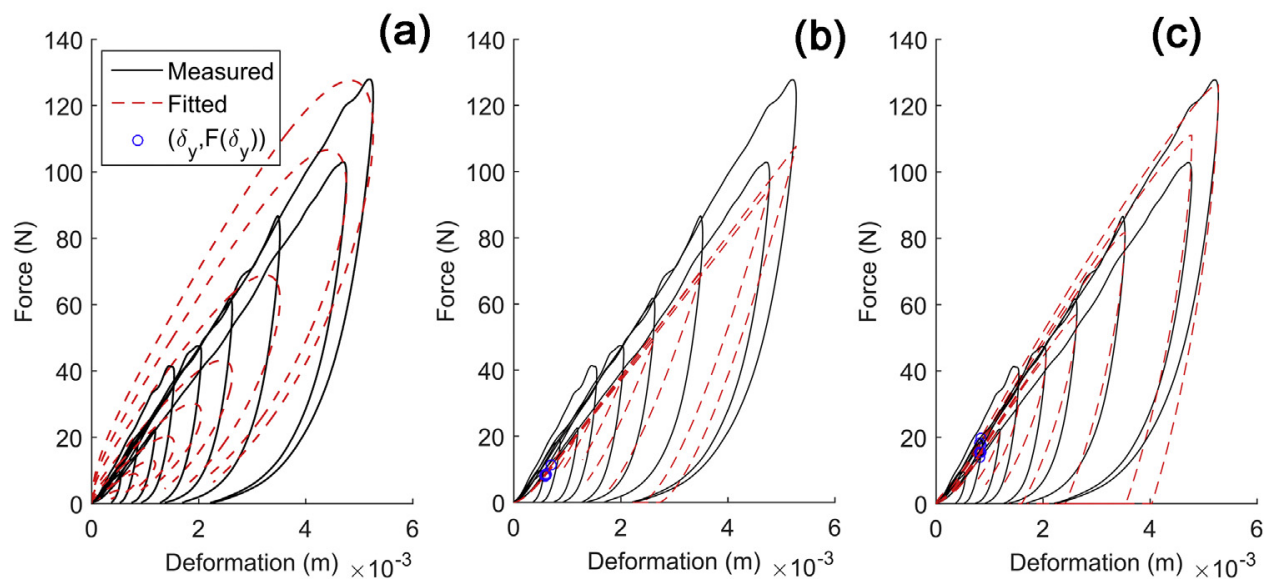

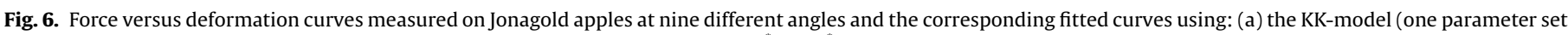

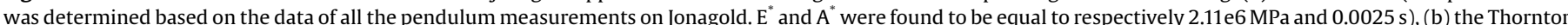

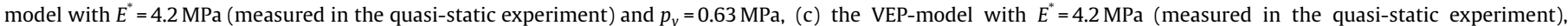

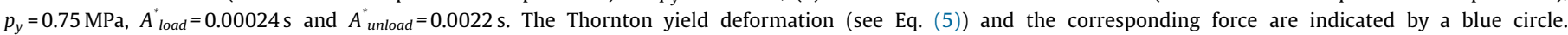




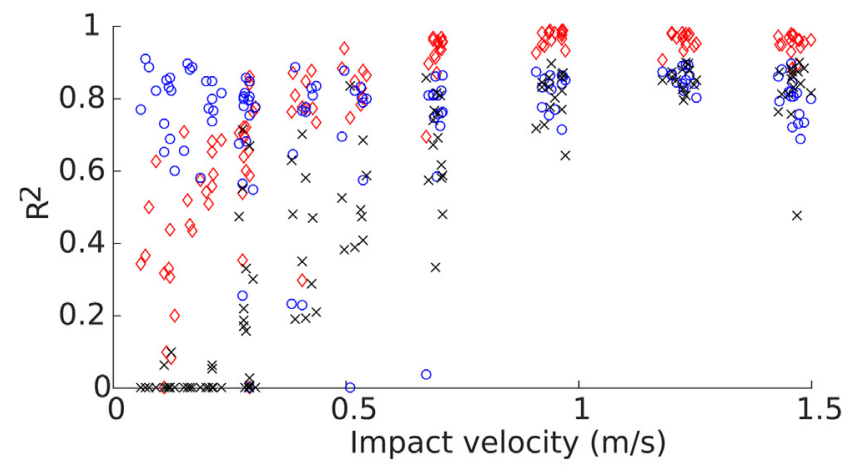

$\diamond$ VEP-model: 1 parameter set per cultivar

- KK-model: parameters that depend on $\dot{\delta}_{i m p}$

$\times$ KK-model: 1 parameter set per cultivar

Fig. 8. $\mathrm{R}^{2}$ value for the force as a function of the impact velocity of each measurement, whereby the force is modelled by the VEP-model with one parameter set per cultivar, the KK-model with velocity dependent parameters and the KK-model with one parameter set per cultivar.

$\dot{\delta}_{i m p}<0.3 \mathrm{~m} / \mathrm{s}$ and $\dot{\delta}_{i m p}>0.3 \mathrm{~m} / \mathrm{s}$ ). In Fig. 8 the $\mathrm{R}^{2}$ value for the force as a function of the impact velocity is depicted for each measurement whereby the force is modelled by the VEP-model with one parameter set per cultivar, the KK-model with velocity dependent parameters and the KK-model with one parameter set per cultivar. The average $\mathrm{R}^{2}$ values corresponding to the latter three are respectively $0.78 \pm 0.24,0.76 \pm 0.17$ and $0.50 \pm 0.35$. Results of the VEP-model with impact velocity dependent parameters can be found in Appendix E, but are not shown in the main text since it can easily lead to overfitting, and prevents the interpretation of the parameters as effective material properties.

According to Fig. 6c, plastic deformation should have occurred in case the force in the measurements became greater than $\pm 20 \mathrm{~N}$ (critical force was calculated per half apple using Eq. (8) with $\delta$ equal to $\delta_{y}$ (Eq. (5)), whereby $\dot{\delta}$ was taken equal to the deformation rate that was measured when the deformation equalled $\delta_{y}$ ). This is confirmed by the observation that small bruises could occur in apples for a maximum force of approximately $20 \mathrm{~N}$. In Table 3, the estimated yield pressure $p_{y}$ and the equivalent dissipative parameters $A^{*}$ load and $A^{*}$ unload are shown for the three different cultivars. Kanzi apples were found to be able to withstand higher pressures than the other two cultivars, while Joly Red apples have the lowest yield pressure. This, however, does not imply that Kanzi apples are less susceptible to bruise damage, because they also have the highest equivalent Young's modulus and, therefore, sustain higher pressures than Jonagold and Joly Red cultivar apples for the same impact.

\subsection{Limitations of the VEP-model}

Bruising of biological tissue is a very complex phenomenon. Although this work can deliver a contribution to the understanding

Table 3

Estimated $p_{y}, \mathrm{~A}_{\text {load }}^{*}$ and $\mathrm{A}_{\text {unload }}^{*}$ per cultivar. During the parameter estimation $\mathrm{E}^{*}$ was fixed to the value estimated from the quasi static compression test (see Table 1).

\begin{tabular}{llll}
\hline & $\mathrm{p}_{\mathrm{y}}(\mathrm{MPa})$ & $\mathrm{A}_{\text {load }}^{*}(\mathrm{~s})$ & $\mathrm{A}_{\text {unload }}^{*}(\mathrm{~s})$ \\
\hline Jonagold & 0.75 & 0.00024 & 0.0022 \\
Joly Red & 0.63 & 0.00018 & 0.0015 \\
Kanzi & 0.83 & 0.00015 & 0.0011 \\
\hline
\end{tabular}

of bruising in apples and other agricultural products, we acknowledge that our model does not take into account all factors that have an influence on the pressures experienced by an apple during bruising and on the resulting bruise damage. One of the limitations of our model is that it does not take into account the effect of apple tissue fatigue that might occur during transport due to repetitive loading. Secondly, we acknowledge the limitation that the presented model is a macroscopic model that assumes homogeneous and isotropic material properties. However, due to differences in cell arrangement and architecture in different zones of the fruit, apple tissue is inhomogeneous and the properties depend on the loading direction (Abbott and $\mathrm{Lu}$, 1996). A model that also contains information at microscopic (i.e. cellular) level could enhance a better understanding of bruising.

\section{Conclusions}

In this study, a visco-elastoplastic contact force model was elaborated and experimentally validated. To that end, measurements of static and dynamic material properties of Jonagold, Kanzi, and Joly Red apple cultivars have been performed. The relatively new cultivar Kanzi was found to be significantly stiffer than the other cultivars. The proposed model is a variant of the elastoplastic Thornton model which can capture the plastic dissipation without a dependency on impact velocity, i.e. it uses true material properties. Even with one parameter set for a large range of degrees of impact the VEP-model delivers reasonable fits of the force curves $\left(R^{2}=0.90 \pm 0.13\right.$ for $\left.\dot{\delta}_{i m p}>0.3 \mathrm{~m} / \mathrm{s}\right)$, considering the complexity and variability of apple tissue.

In the future, the proposed VEP-model could be used to optimise sorting lines, picking robots, transport (e.g. via improved vehicle suspension design) and packaging materials, which could significantly reduce apple waste due to damage. The first step towards this simulation-based optimisation of the apple-handling chain will be to verify whether bruising can be predicted by hypothesizing that damage occurs above the yield pressure measured in this work.

\section{Acknowledgements}

The authors gratefully acknowledge the Flanders Centre of Postharvest Technology (VCBT) for the provision and storage of the apples. Furthermore, we thank the European Union's Seventh Framework Program for research technological development and demonstration through the PicknPack (311987) project and the Agency for Innovation by Science and Technology in Flanders (IWT) through the Chameleon (SB-100021) project and grant no. 111504. At last, we want to thank Agneta Colda, Arno Strouwen, Bram Roosen and Thomas Deckers for carrying out preliminary UTS-experiments.

\section{Appendix A. Checking equality of pressure- and force-based formulations.}

To validate the pressure-based formulations with the forcebased formulation being the reference, simulations with a perfect sphere and a meshed sphere (1280 triangles) were carried out, using respectively the force-based and the pressure-based formulation of the models. A UTS simulation where a flat plane indents a sphere was used to validate the elastoplastic Thornton model, whereas a pendulum simulation was carried out in case of the visco-elastoplastic variant of the Thornton model. Fig. A1 shows that both formulations (i.e. force- and pressured based formulations) of the VEP-model and the Thornton model closely correspond to each other. Only during the unloading phase there is 
(a) Quasi-static simulations

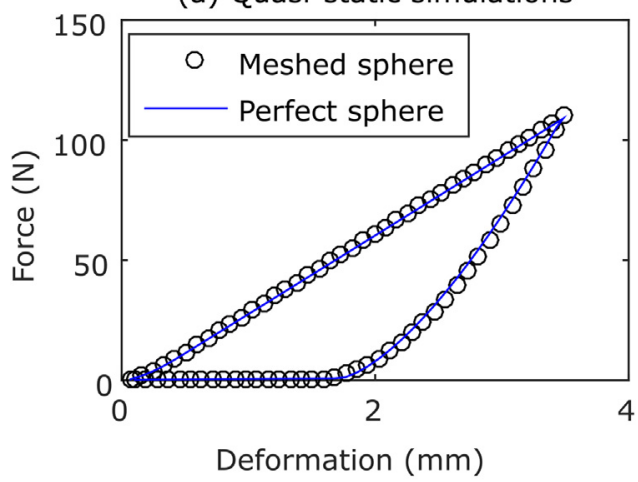

(b) Dynamic simulations

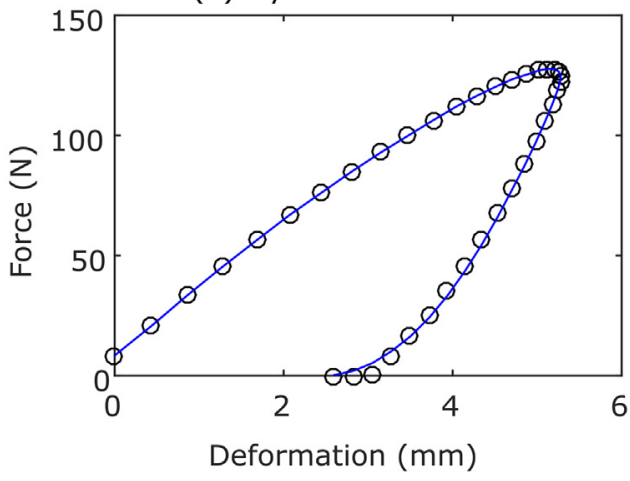

Fig. A1. Loading and unloading curve of analytical model of Thornton and the pressure based formulation, integrated over rounded triangles. A simulation of a plate indenting a sphere using an elastoplastic model (a) and a simulation of pendulum impacting a sphere using a visco-elastoplastic model (b).

a small deviation which is caused by the approximation of the maximal pressure.

\section{Appendix B. Overview of impact levels used in the dynamic experiment.}

See Table B1.

\section{Appendix C. Analysis of quasi-static force-deformation curves.}

To further verify whether the chosen fitting region, as explained in Section 2.3 and shown in Section 3.1, is justifiable, the logarithmic force-deformation curves were investigated. If the elastic Hertz theory is applicable, the force-deformation curve

\section{Table B1}

Overview of the nine impact levels (angle, impact velocity and impact energy) used in the dynamic experiment.

\begin{tabular}{llll}
\hline Level & Angle $\left(^{\circ}\right)$ & $\dot{\delta}_{\text {imp }}(\mathrm{m} / \mathrm{s})$ & $E_{\text {imp }}(\mathrm{J})$ \\
\hline 1 & $\cong 3$ & $\cong 0.12$ & $\cong 0.003$ \\
2 & $\cong 5$ & $\cong 0.21$ & $\cong 0.008$ \\
3 & $\cong 10$ & $\cong 0.28$ & $\cong 0.014$ \\
4 & $\cong 13$ & $\cong 0.42$ & $\cong 0.030$ \\
5 & $\cong 7$ & $\cong 0.53$ & $\cong 0.046$ \\
6 & $\cong 17$ & $\cong 0.69$ & $\cong 0.081$ \\
7 & $\cong 23$ & $\cong 0.95$ & $\cong 0.148$ \\
8 & $\cong 28$ & $\cong 1.23$ & $\cong 0.243$ \\
9 & $\cong 35$ & $\cong 1.46$ & $\cong 0.338$ \\
\hline
\end{tabular}

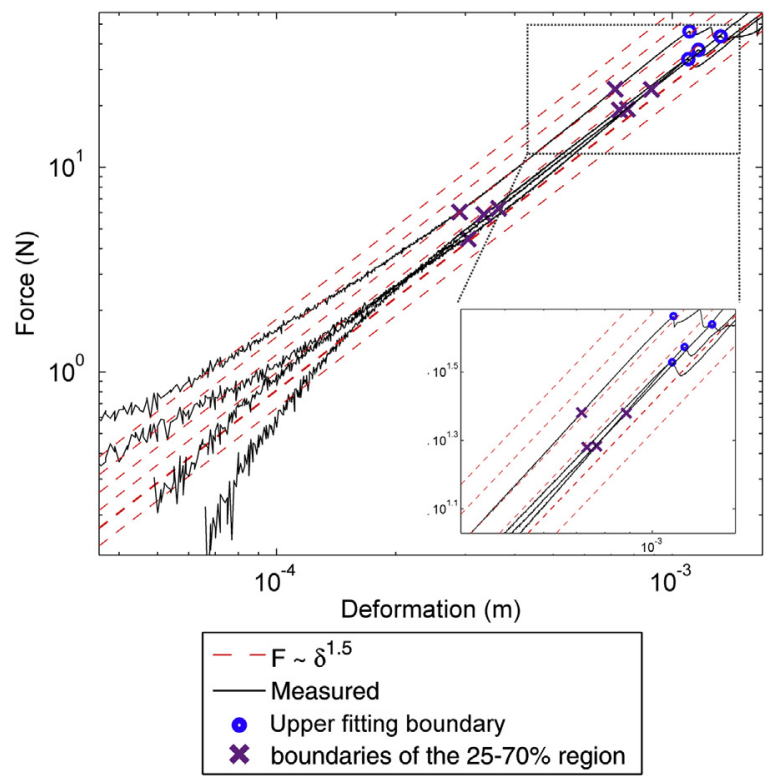

Fig. C1. Four measured quasi-static force-deformation curves plotted on a logarithmic scale. Red lines with slope 1.5 are depicted to indicate the appropriateness of Hertz theory.

should follow a power law with exponent 1.5. Fig. C1 shows a logarithmic plot of four force-deformation curves with the deformation corrected by the extra estimated parameter, as explained in Section 2.3. The first part of the curves do not follow a power law with exponent 1.5 , but this is only small part of the data and the deviation can be attributed to a small error in the determination of the beginning of contact.

The main part of the used fitting region follows a power law with an exponent of approximately 1.5 . To statistically verify this, the following model was fitted to the thirty curves up to the point where the force decreased:

$\log (\mathrm{F})=\mathrm{a} \log (\delta)+\mathrm{b}$

A t-test at 5\% significance level did not reject the null hypothesis that the force-deformation curves have an average slope of 1.5 $(\mathrm{p}=0.469)$.

To check whether a smaller fitting region in the part with a constant power law exponent would yield similar $E^{*}$-values, Hertz' law was fitted on the data with the first $25 \%$ and last $30 \%$ of the data points removed (boundaries of this adapted fitting region are also shown in Fig. (1). These smaller fitting regions resulted in estimated $E^{*}$-values of $4.22 \pm 1.06,5.17 \pm 1.36$ and $8.07 \pm 0.51 \mathrm{MPa}$ for respectively Jonagold, Joly Red and Kanzi apples. Comparison with the $E^{*}$-values in Table 1 , shows that slightly larger average $E^{*}$ values and standard deviations were obtained using the smaller fitting regions. However, the UTS results can still be considered as relatively robust to differences in fitting boundaries.

Table D1

$\mathrm{E}^{*}, p_{y}, \mathrm{~A}_{\text {load }}^{*}$ and $\mathrm{A}_{\text {unload }}^{*}$ determined per cultivar by fitting the VEP model to the experimental impact data.

\begin{tabular}{lllll}
\hline & $\mathrm{E}^{*}(\mathrm{MPa})$ & $\mathrm{A}_{\text {load }}^{*}(\mathrm{~s})$ & $\mathrm{A}_{\text {unload }}^{*}(\mathrm{~s})$ & $p_{y}(\mathrm{MPa})$ \\
\hline Jonagold & 3.67 & 0.00032 & 0.0028 & 0.78 \\
Joly Red & 4.10 & 0.00023 & 0.0022 & 0.65 \\
Kanzi & 6.85 & 0.00018 & 0.0015 & 0.84 \\
\hline
\end{tabular}


Table E1

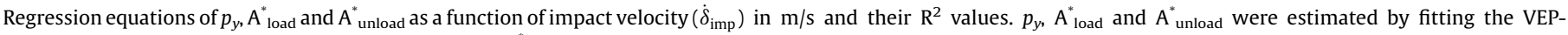
model to the measured force-deformation curves ( $E^{*}$ equal to those presented in Table 1 ).

\begin{tabular}{|c|c|c|c|c|c|c|}
\hline & $\mathrm{A}_{\text {load }}^{*}=$ & $\mathrm{R}^{2}$ & $\mathrm{~A}_{\text {unload }}^{*}=$ & $\mathrm{R}^{2}$ & $p_{y}=$ & $\mathrm{R}^{2}$ \\
\hline Jonagold & $0.00018\left(\dot{\delta}_{\text {imp }}\right)^{-1.47}$ & 0.86 & $0.0027\left(\dot{\delta}_{\mathrm{imp}}\right)^{-0.38}$ & 0.33 & $0.76 \mathrm{e} 5 \dot{\delta}_{\mathrm{imp}}+6.97 \mathrm{e} 5$ & 0.092 \\
\hline Joly Red & $0.000055\left(\dot{\delta}_{\text {imp }}\right)^{-1.45}$ & 0.62 & $0.0019\left(\dot{\delta}_{\text {imp }}\right)^{-0.78}$ & 0.61 & $0.23 \mathrm{e} 5 \dot{\delta}_{\mathrm{imp}}+6.47 \mathrm{e} 5$ & 0.009 \\
\hline Kanzi & $0.00017\left(\dot{\delta}_{\mathrm{imp}}\right)^{-0.89}$ & 0.78 & $0.0014\left(\dot{\delta}_{\mathrm{imp}}\right)^{-0.59}$ & 0.65 & $0.09 \mathrm{e} 5 \dot{\delta}_{\mathrm{imp}}+8.50 \mathrm{e} 5$ & 0.001 \\
\hline
\end{tabular}

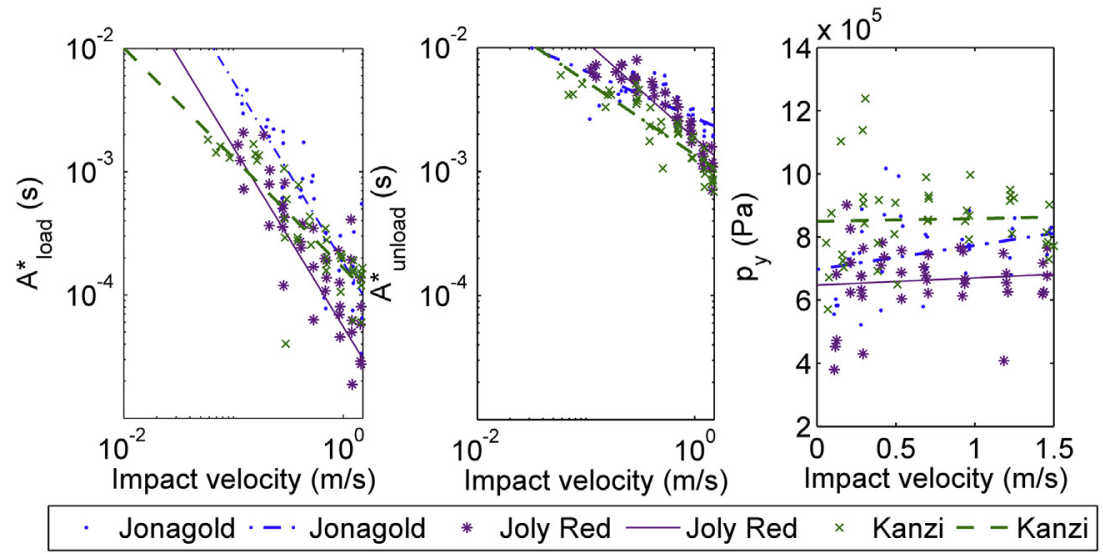

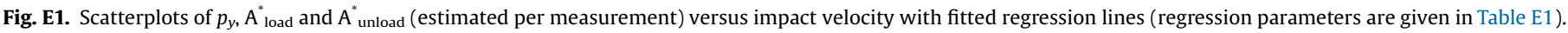

Table E2

Average $\mathrm{R}^{2}$ values of the VEP- and KK-model with or without velocity dependency of respectively the parameters $p_{y}, \mathrm{~A}^{*}$ load and $\mathrm{A}^{*}$ unload and the parameters $A^{*}$ and $E^{*}$.

\begin{tabular}{|c|c|c|c|c|}
\hline & \multicolumn{2}{|l|}{$\underline{\text { VEP-model }}$} & \multicolumn{2}{|l|}{ KK-model } \\
\hline & $\dot{\delta}_{\text {imp }}$ dependent parameters & $\dot{\delta}_{\text {imp }}$ independent parameters & $\dot{\delta}_{\text {imp }}$ dependent parameters & $\dot{\delta}_{\text {imp }}$ independent parameters \\
\hline $\mathrm{R}^{2}$ for impacts with $\dot{\delta}_{\text {imp }}<0.3 \mathrm{~m} / \mathrm{s}$ & $0.86 \pm 0.15$ & $0.51 \pm 0.22$ & $0.74 \pm 0.18$ & $0.11 \pm 0.20$ \\
\hline $\mathrm{R}^{2}$ for impacts with $\dot{\delta}_{\mathrm{imp}}>0.3 \mathrm{~m} / \mathrm{s}$ & $0.94 \pm 0.12$ & $0.90 \pm 0.13$ & $0.76 \pm 0.18$ & $0.71 \pm 0.20$ \\
\hline $\mathrm{R}^{2}$ for all impacts & $0.91 \pm 0.13$ & $0.78 \pm 0.24$ & $0.76 \pm 0.17$ & $0.50 \pm 0.35$ \\
\hline
\end{tabular}

\section{Appendix D. Four-parametric fit of the VEP model.}

Whereas the parameters in Table 3 were obtained by a threeparametric fit $\left(p_{y}, \mathrm{~A}_{\text {load }}^{*}\right.$ and $\left.\mathrm{A}_{\text {unload }}^{*}\right)$ of the VEP model on the impact data, the parameters presented in Table D1 resulted from a four-parametric fit $\left(\mathrm{E}^{*}, p_{y}, \mathrm{~A}_{\text {load }}^{*}\right.$ and $\left.\mathrm{A}_{\text {unload }}^{*}\right)$. The three $\mathrm{E}^{*}$-values in Table D1 are relatively close to the $\mathrm{E}^{*}$-values obtained from the quasi-static compression experiment (see Table 1 ), which indicates consistency between both experiments.

\section{Appendix E. VEP-model with impact velocity dependent parameters.}

In this section a different $p_{y}, A_{\text {load }}^{*}$ and $A_{\text {unload }}^{*}$ for each measurement was estimated and afterwards for each of these three parameters a regression equation was constructed that predicts the parameter value in function of impact velocity. The relation between impact velocity and the parameters the VEPmodel with velocity dependent $p_{y}, \mathrm{~A}^{*}$ load and $\mathrm{A}_{\text {unload }}^{*}$ parameters can be seen in Table E1 and Fig. E1. Note that the dissipative parameters decrease with increasing impact velocity in a power law fashion. $p_{y}$ slightly increases with increasing impact velocity, which could correspond to the observation of increasing failure strain and failure stress with increasing strain rate made by Baritelle and Hyde (2001). However, this velocity-effect of $p_{y}$ is very small (slope/intercept-ratio between 0.01 and 0.1 ) and the $\mathrm{R}^{2}$ is low. The $\mathrm{R}^{2}$-values corresponding to the VEP-model with impact velocity dependent parameters are shown in Table E2 and Fig. E2.

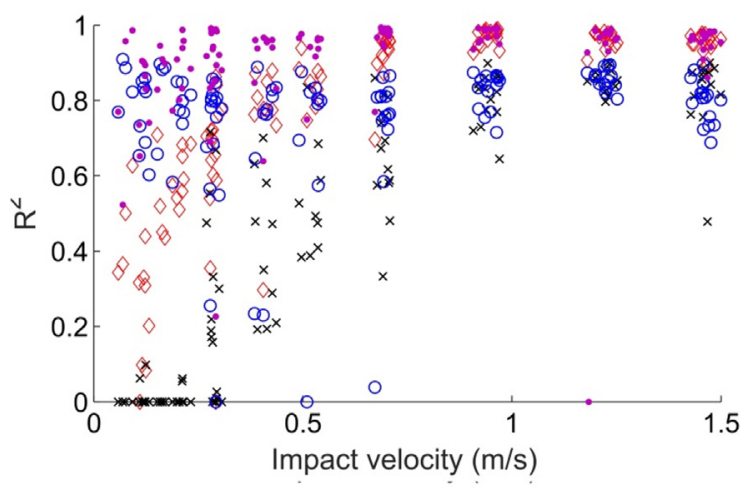

$\checkmark$ VEP-model: 1 parameter set per cultivar
$\times \quad$ VEP-model: parameters that depend on $\dot{\delta}_{i m p}$
$\times \quad$ KK-model: 1 parameter set per cultivar
$\circ$ KK-model: parameters that depend on $\dot{\delta}_{i m p}$

Fig. E2. Fig. 8 supplemented with the $\mathrm{R}^{2}$ values related to the VEP-model with velocity dependent parameters (see Table E1). 
For both $\dot{\delta}_{\text {imp }}<0.3 \mathrm{~m} / \mathrm{s}$ and $\dot{\delta}_{\text {imp }}>0.3 \mathrm{~m} / \mathrm{s}$, the VEP-model with impact velocity dependent parameters has a higher average $\mathrm{R}^{2}$ value than the other three options (i.e. VEP- and KK-model with one parameter set per cultivar and KK-model with impact velocity dependent parameters). However, better fits on the pendulum data by including velocity dependency of the parameters into the model, do not guarantee that the model would also be physically more correct.

\section{References}

Abbott, J.A., Lu, R., 1996. Anisotropic mechanical properties of apples. Trans. ASAE 39, 1451-1459.

Ahmadi, E., Ghassemzadeh, H.R., Sadeghi, M., Moghaddam, M., Neshat, S.Z., Ettefagh, M.M. 2012. Dynamic modeling of peach fruit during normal impact. J. Food Process Eng. 35, 483-504. doi:http://dx.doi.org/10.1111/j.17454530.2010.00603.x.

Arnold, P.C., Mohsenin, N.N., 1971. Proposed techniques for axial compression tests on intact agricultural products of convex shape. Trans. ASABE 14, 78-84.

ASAE Standard, 1998. Compression Test of Food Materials of Convex Shape. Am. Soc. Agric. Eng. S368.3, pp. 580-587.

Baritelle, A.L., Hyde, G.M., 2001. Commodity conditioning to reduce impact bruising. Postharvest Biol. Technol. 21, 331-339.

Brilliantov, N.V., Spahn, F., Hertzsch, J.M., Pöschel, T., 1996a. A model for collisions in granular gases. Am. Phys. Soc. 53, 11. doi:http://dx.doi.org/10.1103/ PhysRevE.53.5382.

Brilliantov, N.V., Spahn, F., Hertzsch, J.M., Pöschel, T., 1996b. The collision of particles in granular systems. Phys. A Stat. Mech. Appl. 231, 417-424. doi:http://dx.doi. org/10.1016/0378-4371(96)00099-4.

Coleman, T.F., Li, Y., 1996. An interior trust region approach for nonlinear minimization subject to bounds. SIAM J. Optim. 6, 418-445. doi:http://dx.doi. org $/ 10.1137 / 0806023$

Herold, B., Geyer, M., Studman, C.J., 2001. Fruit contact pressure distributionsequipment. Comput. Electron. Agric. 32, 167-179.

Knee, M., Miller, A.R., 2002. Mechanical damage. Fruit Quality and Its Biological Basis. Sheffield Academic Press, Sheffield pp. 157-179.
Kruggel-Emden, H., Wirtz, S., Scherer, V., 2009. Applicable contact force models for the discrete element method: the single particle perspective. J. Press. Vessel Technol. 131, 024001. doi:http://dx.doi.org/10.1115/1.3040682.

Kuwabara, G., Kono, K., 1987. Restitution coefficient in a collision between two spheres. Jpn. J. Appl. Phys. 26, 1230-1233.

Lewis, R., Yoxall, A., Marshall, M.B., Canty, L.A., 2008. Characterising pressure and bruising in apple fruit. Wear 264, 37-46.

Mohsenin, N.N., 1986. Physical Properties of Plant and Animal Materials: Structure, Physical Characteristics and Mechanical Properties. Gordon and Breach, New York (N.Y.).

Rathbone, D., Marigo, M., Dini, D., van Wachem, B., 2015. An accurate forcedisplacement law for the modelling of elastic-plastic contacts in discrete element simulations. Powder Technol. 282, 2-9. doi:http://dx.doi.org/10.1016/j. powtec.2014.12.055.

Smeets, B., Odenthal, T., Keresztes, J., Saeys, W., 2014. Modeling arbitrary curved shapes in the Discrete Element Model using triangulated rounded bodies.

Thornton, C., Ning, Z., 1998. A theoretical model for the stick/bounce behaviour of adhesive: elastic-plastic spheres. Powder Technol. 99, 154-162.

Tijskens, E., Ramon, H., Baerdemaeker, J.D., 2003. Discrete element modelling for process simulation in agriculture. J. Sound Vib. 266, 493-514. doi:http://dx.doi org/10.1016/S0022-460X(03)00581-9.

Van Zeebroeck, M., 2005. The Discrete Element Method (DEM) to Simulate Fruit Impact Damage During Transport and Handling. KU Leuven.

Van Zeebroeck, M., Tijskens, E., Dintwa, E., Kafashan, J., Loodts, J., De Baerdemaeker, J., Ramon, H., 2006. The discrete element method (DEM) to simulate fruit impact damage during transport and handling: model building and validation of DEM to predict bruise damage of apples. Postharvest Biol. Technol. 41, 85-91.

Vu-Quoc, L., Zhang, X., Lesburg, L., 2000. A normal force-displacement model for contacting spheres accounting for plastic deformation: force-driven formulation. J. Appl. Mech. 67, 363. doi:http://dx.doi.org/10.1115/1.1305334.

Zarifneshat, S., Ghassemzadeh, H.R., Sadeghi, M., Abbaspour-Fard, M.H., Hamid, E. Javadi, A., Shervani-Tabar, M.T., 2010. Effect of impact level and fruit properties on golden delicious apple bruising. Am. J. Agric. Biol. Sci. 5, 114-121.

Zhang, X., Vu-Quoc, L., 2002. A method to extract the mechanical properties of particles in collision based on a new elasto-plastic normal force-displacement model. Mech. Mater. 34, 779-794. doi:http://dx.doi.org/10.1016/S0167-6636 (02)00181-3. 